\title{
RESET
}

Recherches en sciences sociales sur Internet

5 | 2016

La critique culturelle : déclin ou hégémonie?

\section{La critique littéraire amateur sur les blogs de lecteurs}

Une concurrence limitée de la critique littéraire professionnelle Amateur literary criticism on readers' blogs versus professional literary criticism: a competition of limited importance

\section{Géraldine Bois, Emilie Saunier et Olivier Vanhée}

\section{(2) OpenEdition}

Journals

Édition électronique

URL : http://journals.openedition.org/reset/736

DOI : $10.4000 /$ reset.736

ISSN : 2264-6221

Éditeur

Association Recherches en sciences sociales sur Internet

Référence électronique

Géraldine Bois, Emilie Saunier et Olivier Vanhée, «La critique littéraire amateur sur les blogs de lecteurs », RESET [En ligne], 5 | 2016, mis en ligne le 30 juin 2016, consulté le 14 novembre 2019. URL http://journals.openedition.org/reset/736; DOI : 10.4000/reset.736

Ce document a été généré automatiquement le 14 novembre 2019.

(c) Association Recherches en sciences sociales sur Internet 


\title{
La critique littéraire amateur sur les blogs de lecteurs
}

\author{
Une concurrence limitée de la critique littéraire professionnelle \\ Amateur literary criticism on readers' blogs versus professional literary \\ criticism: a competition of limited importance
}

Géraldine Bois, Emilie Saunier et Olivier Vanhée

\section{Introduction}

1 Les dispositifs de jugement en ligne rendent visibles les évaluations profanes dans de nombreux secteurs culturels et commerciaux ${ }^{1}$. Cet article évoque l'un de ces dispositifs dans le secteur de la littérature: les blogs de critique littéraire "amateur", souvent appelés «blogs de lecteurs» par les professionnels du livre (éditeurs, critiques littéraires, libraires, bibliothécaires), qui se sont développés à partir du début des années 2000. Spécialisés dans la publication des commentaires de livres lus par les auteurs de ces blogs, ils sont à distinguer des blogs tenus par des «professionnels » de la critique littéraire «traditionnelle » (c'est-à-dire la critique présente dans des titres de presse imprimée ou des émissions de télévision et de radioº ${ }^{2}$ ). En tant que supports d'auto-publication accordant une large place aux commentaires rédigés, les blogs de lecteurs se distinguent aussi des dispositifs «d'agrégation des notes et des avis des consommateurs" (Beauvisage et al., 2014), «qui proposent de concentrer les contributions et de les synthétiser algorithmiquement » (Cardon, 2014).

2 Les blogs de lecteurs témoignent, dans un domaine traditionnellement dominé par la critique professionnelle de la presse ou des médias audiovisuels, d'une diversification des émetteurs de jugements publics sur les livres permise par Internet. Caractérisés par un faible coût d'entrée et une capacité à toucher un public très large, ces blogs bousculent le modèle communicationnel traditionnel, qui impliquait un nombre limité d'émetteurs experts et une masse de récepteurs profanes (Cardon, 2010; Auray \& Moreau, 2012), en faisant exister « des individus tour à tour émetteurs et récepteurs » 
(Cardon, $2010:$ 8). Dans ce contexte, où Internet fait « appar(aître) des amateurs sur la scène publique » et « ôte le privilège d'accès à la publication dont bénéficiaient naguère les professionnels » (Cardon, $2010: 9$ ), assiste-t-on en pratique à un renouvellement des formes de la critique ayant le pouvoir de promouvoir et de consacrer des titres de littérature ? Dans quelle mesure la critique littéraire professionnelle traditionnelle estelle concurrencée par la critique littéraire amateur en ligne?

Plusieurs travaux récents soulignent les limites de la «démocratisation» que permettrait l'évaluation en ligne des biens culturels: la visibilité sur Internet ne subvertit pas les modes traditionnels de prescription et les hiérarchies culturelles établies (Bastard et al., 2012 ; Beaudoin \& Pasquier, 2014; Beauvisage et al., 2014; Benhamou et al., 2012 ; Beuscart \& Couronné, 2009). L'hypothèse selon laquelle la vente et la consommation de produits culturels sur Internet seraient marquées par une plus grande diversité que dans les "réseaux physiques» de distribution a été nuancée, notamment dans le domaine de la vente de livres (Bounie et al., 2010 ; Moreau \& Peltier, 2011). Si d'autres enquêtes abordent plus précisément les rapports entre "critique professionnelle » (dans les «médias traditionnels », parfois sur des supports en ligne) et « critique amateur» (sur des blogs, des sites marchands ou des plateformes dédiées), elles concernent d'autres domaines que la littérature. Anne-Sophie Béliard étudie ainsi "la place des blogs d'évaluation dans les circuits de consécration des séries et la position des amateurs-blogueurs dans l'espace de la critique sériephile, notamment leur rapport aux professionnels» (Béliard, $2014: 96)$. Si elle attire l'attention sur la porosité des frontières entre amateurs et professionnels, c'est en partie parce que la critique de séries télévisées est un domaine émergent dont l'« institutionnalisation et [la] professionnalisation restent en construction ", ce qui la distingue de la critique littéraire, mais aussi en raison du choix d'enquêter auprès de blogueurs amateur appartenant à «la frange experte » des blogueurs, qui «explicitent des ambitions critiques» (Béliard, 2014: 98). Dans le domaine plus institutionnalisé des critiques cinématographiques, Valérie Beaudouin et Dominique Pasquier notent que « la critique amateur de film, loin de proposer un modèle alternatif et concurrent de classement par le jugement profane, renforce in fine le modèle du jugement expert» (Beaudoin \& Pasquier, 2014 : 157). Des enquêtes par questionnaires sur les «motivations» des blogueurs soulignent par ailleurs que "l'évaluation », ou la volonté de peser face à la "critique professionnelle", ne figurent pas au premier rang et que les blogs remplissent bien d'autres fonctions (Naulin, 2014 ; Pinch \& Kesler, 2011).

4 Si le présent article s'intéresse au même type d'acteurs que ces enquêtes (les critiques amateur en ligne, les critiques professionnels et les professionnels de l'univers culturel considéré), il traite plus spécifiquement et dans le détail des rapports de force entre ces deux instances (critique amateur et critique professionnelle) du point de vue de leur pouvoir de promotion. Il montre que la concurrence entre ces deux formes de critiques reste en réalité limitée et qu'on a davantage affaire à une coprésence de deux formes de critiques - c'est-à-dire une existence de la critique amateur à côté de la critique professionnelle - plutôt qu'à une concurrence entre elles. Il nuance ainsi la représentation du Web comme un espace d'émergence de «contre-pouvoirs ou de systèmes de valeurs parallèles » (Couleau \& Hellegouarch, $2010: 7-8$ ), ou encore comme un lieu propice à la "montée en puissance des amateurs" qui remettrait en cause l'élitisme et le pouvoir des autorités culturelles traditionnelles (et professionnelles), en 
accordant de la visibilité et une voix sur la scène publique à des «homme(s) ordinaire(s)» (Flichy, $2010: 11)$.

L'analyse des positionnements entre critiques professionnels et blogueurs amateur est ici menée au travers de deux perspectives différentes. Dans un premier temps, l'article interroge le rôle que les acteurs traditionnels de l'univers littéraire - et en particulier les éditeurs - attribuent aux blogs de lecteurs par rapport à la critique professionnelle. Il étudie ainsi les manières de faire et de voir d'acteurs dont les pratiques professionnelles influencent directement l'état des rapports de force entre les deux formes de critiques : si les éditeurs - et en particulier les plus importants d'entre eux se tournaient massivement vers les blogueurs plutôt que vers les critiques professionnels, cela signifierait sans aucun doute que les premiers auraient gagné en influence et en légitimité. Sur ce point, l'article montre que l'existence des blogs ne bouleverse pas les pratiques de promotion des livres des éditeurs ni leurs perceptions des hiérarchies entre critique amateur et critique professionnelle. Dans un second temps, la question du rapport de force entre blogueurs et critiques professionnels est abordée au travers du rôle que les blogueurs eux-mêmes s'attribuent dans le cadre de leur activité de commentaire des livres. Il s'agit plus précisément d'étudier la place qu'ils s'accordent dans la promotion des livres ainsi que leurs prétentions à jouer sur le même terrain que la critique professionnelle. En effet, pour qu'il y ait concurrence, il faut que les parties en présence agissent et se pensent les unes par rapport aux autres. Si nous n'avons pas analysé en détail la manière dont les critiques professionnels se positionnent par rapport aux blogueurs ${ }^{3}$, la prétention de ces derniers à renverser les hiérarchies établies est en revanche au centre de notre étude. À ce sujet, l'article montre que rares sont les blogueurs qui pensent avoir un pouvoir déterminant dans la promotion des livres et qui se positionnent comme des concurrents de la critique professionnelle. Ainsi, l'état de la concurrence entre les deux formes de critiques s'apprécie non seulement au travers du point de vue des éditeurs, qui leur accordent plus ou moins d'importance lors de leur travail promotionnel, mais aussi au travers du point de vue des blogueurs eux-mêmes, qui agissent et se vivent plus ou moins comme des concurrents des instances de promotion et de jugement littéraires établies, et qui contribuent ainsi à faire exister à des degrés très divers cet état de concurrence.

\section{Méthodologie}

Afin d'analyser la prise en compte des blogs de lecteurs par les acteurs traditionnels de l'univers littéraire, vingt entretiens ont été réalisés avec des professionnels du livre (libraires, éditeurs, organisateurs de manifestation littéraire, etc.) et des écrivains. Cet article s'appuiera en particulier sur les matériaux recueillis auprès de plusieurs personnes travaillant dans des maisons d'éditions (directeurs, attachés de presse et responsables numériques), qui se sont avérées les acteurs les plus concernés par les blogs de lecteurs, même s'ils le sont inégalement. Les sept éditeurs interrogés ont des propriétés variées, du point de vue de leur degré de légitimité littéraire (certains d'entre eux ont une forte légitimité littéraire alors que d'autres sont des éditeurs commerciaux de divertissement) et du point de vue de leur taille (notre population d'éditeurs s'étend du micro-éditeur régional auto-diffusé au grand éditeur national ${ }^{4}$ ). L'objectif de ces entretiens était de saisir leurs usages et leurs perceptions des blogs et de les comparer à leurs usages et à leurs perceptions de la presse et des 
médias audiovisuels. Le phénomène des blogs est en outre suffisamment ancien pour que ces professionnels du livre puissent témoigner avec un peu de recul de ce qu'il a changé dans leurs pratiques et leurs représentations de la promotion des livres de littérature.

Un deuxième volet de la recherche a consisté à mener une analyse approfondie de quatre blogs : L'Anagnoste, Biblioblog, Les Livres de George et un blog dont la responsable, "Catherine ", a souhaité qu'il reste anonyme, et que nous avons nommé « Manon et les livres ». Nous avons réalisé des entretiens avec les responsables de ces quatre blogs (nommés respectivement Éric Bonnargent, Laurence, George et Catherine ${ }^{5}$ ) et analysé parallèlement leur contenu (en particulier les billets que leurs auteurs y publient ${ }^{6}$ ). Ces blogueurs ont été choisis parce qu'ils avaient en commun de tenir des blogs actifs (ils postent de une à cinq critiques par semaine) et relativement anciens, puisque les quatre blogs ont été créés entre 2005 et 2008. Dans le champ littéraire, durer constitue un signe distinctif associé à la capacité à « se faire un nom » (Bourdieu, 1998 : 262). Ce critère de reconnaissance symbolique s'applique au cas d'Internet où un grand nombre de blogs ou de sites se créent et disparaissent rapidement. Ces deux critères (ancienneté, activité) ont été choisis car ils assuraient à ces blogs une visibilité qui augmentait leurs chances d'être repérés par des acteurs traditionnels de l'univers littéraire et de prendre position par rapport à eux. Ainsi, aucun des blogs choisis n'est confidentiel (les quatre blogueurs interrogés déclarent plusieurs milliers de visiteurs par mois).

Le type de visibilité de ces quatre blogs a été approché en mobilisant une distinction qui fait généralement sens au sein des univers de production culturelle (Bourdieu, 1998) : l'opposition entre la reconnaissance par le « nombre » (large visibilité) et la reconnaissance par la « qualité » (jugement des « experts »). Deux classements dont les critères se rapprochent le plus de ces deux formes de reconnaissance ont été mobilisés : d'un côté, le classement effectué par le site Wikio (où figuraient les blogs de George et de Catherine) sur la base d'un critère quantitatif relatif à «l'influence » des blogs (i. e. le nombre de liens qui pointent vers eux) ; de l'autre, la sélection de blogs disponible dans la rubrique « Signets » du site de la Bibliothèque Nationale de France (blogs d'Éric Bonnargent et de Laurence) et réalisée par des bibliothécaires, c'est-à-dire par des « experts » en matière de littérature, en fonction de critères davantage liés à la qualité du contenu des blogs?

\section{Du côté des éditeurs : une mise en concurrence très limitée}

L'analyse des entretiens avec les éditeurs permet de mettre en évidence quelques déplacements, dus à l'existence des blogs de lecteurs, dans leurs pratiques et représentations de la promotion des livres. En effet, les éditeurs accordent parfois une place effective aux blogueurs. Mais cette place est soit très limitée, soit ne concerne que des petits éditeurs. De plus, ces pratiques de recours aux blogs sont motivées par une croyance dans les capacités promotionnelles et dans le statut spécifique des blogueurs. 
Parce que ces capacités concernent essentiellement la promotion d'autres types de livres ainsi que d'autres manières de les promouvoir et que les blogueurs sont avant tout considérés comme des amateurs, la mise en concurrence entre blogueurs et critiques professionnels par les éditeurs est finalement limitée.

\section{Le recours aux blogs : des pratiques circonscrites}

$7 \quad$ Les éditeurs interviewés se sont progressivement saisis des différents outils de visibilité en ligne et ont en particulier intégré les blogs de lecteurs dans leurs pratiques de promotion des livres 8 . Pour les maisons d'édition de taille «moyenne » ou « grande », il s'agit essentiellement de profiter d'un outil supplémentaire et peu coûteux en temps et en argent. Cela permet d'accroître la visibilité de leurs titres et d'occuper « l'espace médias » comme l'explique un enquêté :

$\mathrm{Y}$ a des nouvelles zones, y a des nouveaux espaces... Donc on va pas les laisser à la concurrence. Donc de la même manière que certaines grosses maisons d'édition vont chercher à avoir toutes les têtes de gondoles dans les librairies ou dans les supermarchés, ils essaient d'occuper l'espace médias. [...] Y a une prise de conscience, on se dit qu'il y a quand même pas mal de monde qui passe du temps en ligne. Donc ça peut être intéressant. (Responsable du développement numérique d'un grand groupe d'édition)

Les blogueurs peuvent également être considérés par ces éditeurs comme «le dernier recours » dans le cas d'une promotion difficile :

Quand je suis euh... à la ramasse sur un livre, que j'ai un auteur qui vient dans trois jours et que j'ai pas l'ombre d'une interview qui se profile pour lui, oui, je vais solliciter les blogs. (Attachée de presse d'une grande maison d'édition commerciale)

Enfin, il s'agit de se saisir des possibilités liées à l'archivage en ligne, permettant selon eux de maintenir des ventes en petite quantité sur un temps long. Les critiques archivées sont en effet accessibles par les moteurs de recherche bien après leur publication initiale :

Un des avantages des blogs aussi, c'est que la trace elle reste. Ça reste sur Internet alors que quand vous faites une pige, après le papier il part à la poubelle et l'information elle reste pas longtemps chaude. Même si elle a tendance à se refroidir sur les blogs, ça reste encore un peu actif quoi. (Responsable numérique d'un grand groupe d'édition)

Cependant, dans la rationalisation de leur temps de travail effectif, on observe que ces éditeurs ciblent prioritairement les médias traditionnels et accordent moins de temps à la communication envers les blogs et d'argent à l'envoi de services de presse à leur attention :

Je ne passe pas de temps avec les blogueurs, très peu, très très peu de temps. Je ne calcule pas mais c'est très peu. Je vous dis, je vais juste vérifier une ou deux choses. [...] L'essentiel pour moi, c'est d'obtenir de la presse des critiques. [...] Voilà. Le plus clair de mon temps et le but de la manœuvre, c'est ça! Ce qui n'exclut pas le reste, c'est-à-dire les blogs, les sites. Mais proportionnellement, c'est pas comparable. Ça ne peut pas l'être. (Attachée de presse d'une maison d'édition de taille moyenne)

Disons que [mes relations avec les blogueurs] restent encore infimes hein ! C'est pas $\mathrm{du}$ tout un enjeu les blogs, [...] ça reste minime... Faut pas se voiler la face. [...] Je n'arrose pas la blogosphère de services de presse quoi ! Déjà j'en ai pas les moyens ni matériels, ni financiers. Et bon, je... je suis obligée de hiérarchiser quand même! (Attachée de presse d'un grand éditeur commercial) 
11 Pour ces éditeurs, les médias classiques sont en effet définis comme ayant, encore aujourd'hui, largement plus de poids que les blogs de lecteurs. Pour l'attachée de presse d'une maison d'édition nationale de taille moyenne, ce poids moindre des blogs est même une "question de bon sens". Elle souligne ainsi que son activité reste majoritairement orientée vers les relations avec ces médias professionnels : «Une page dans Le Monde ou dans Libé ça vaut tous les blogs! Tous les blogs. C'est incomparable! ».

On comprend dans ces conditions que ces éditeurs fassent preuve d'une attitude de déférence plus importante à l'égard des médias et d'une moindre indulgence envers les blogs. Par exemple, l'attachée de presse précédemment citée n'hésite pas à cesser tout partenariat avec un blogueur qu'elle n'estime pas compétent ou pertinent ${ }^{9}$. En revanche, elle n'ose pas le faire dans le cas des médias traditionnels :

La pression n'est pas la même. C'est-à-dire que quand vous cherchez à faire connaître un auteur et à obtenir une critique dans le Monde ou Libé, le Nouvel Obs ou Télérama c'est beaucoup plus difficile. Le blogueur est celui qui vous sollicite. [...] Si je trouve que le type est un crétin absolu, je le supprime simplement de l'envoi de... [livres] Voilà ! [...] [Par exemple] une critique malhonnête sur un livre. Ça serait les Inrocks ou Télérama, j'aurais pas la même attitude. Je dirais: "Écoute, je ne comprends pas ce qui se passe, pourquoi parler comme ça » [...] Je leur dirais ce que je pense ! Sauf que Télérama c'est important alors qu'un blogueur ça ne l'est pas. Donc ça veut dire que Télérama vous diriez quelque chose, mais...

Je serai tenue de leur envoyer au prochain coup. C'est tout! Alors qu'un blog, je suis pas tenue. (Attachée de presse d'une maison d'édition nationale de taille moyenne)

13 Le recours massif aux blogs concerne en fait seulement, au sein de notre population d'éditeurs, les plus petits d'entre eux. Ils s'investissent dans la construction d'une visibilité en ligne faute d'accès aux moyens traditionnels de diffusion et de promotion, que ce soit la présence large en librairies ou la critique littéraire dans la presse et les médias audiovisuels (Legendre \& Abensour, 2007). Pour ces petites maisons d'édition, la consultation et la prise de contact avec les blogs de lecteurs constituent une activité quotidienne :

Je passe beaucoup, beaucoup de temps à aller lire les blogs [...], voir les ouvrages qui ont été chroniqués, les relais éventuellement qui ont été faits autour de nos ouvrages, etc. [...] Donc oui, ça prend du temps. Mais c'est le prix à payer. [...] Enfin moi, je ne le prends pas du tout comme une contrainte, au contraire, j'ai besoin de ça... (Directeur d'une petite maison d'édition régionale associative)

14 Ce sont ces petits éditeurs qui, faisant sans doute de nécessité vertu, expriment le plus une croyance dans le pouvoir promotionnel des blogs. Ils se montrent ainsi particulièrement sensibles aux indicateurs de l'efficacité de ce type de prescription, même si elle semble difficile à vérifier :

On sait très bien que quand [notre dernier ouvrage] est sorti, on a eu un relais absolument incroyable sur la Toile. [...] Et ça, ça a été, on le sait, prescripteur, parce que les ventes ont explosées en mai. [...] Y a eu vraiment une espèce d'énervement en termes de commandes [sourire] et c'est pas venu comme ça par hasard parce qu'il n'était pas encore relayé véritablement sur les librairies. Mais y a eu énormément d'articles sur la blogosphère littéraire qui sont parus et on pense que ça vient de là. Je vois pas d'où ça pourrait venir. (Directeur d'une petite maison d'édition régionale associative)

15 Ainsi, parce que l'usage des blogs et la croyance dans leur pouvoir promotionnel concernent avant tout les petits éditeurs, les rapports de force entre critiques professionnels et blogueurs amateur sont loin d'être bouleversés. En effet, les petits éditeurs, du fait de leur position dominée dans l'espace éditorial, n'ont pas le pouvoir 
de légitimer une nouvelle forme de critique face aux critiques établies. Si les pratiques des éditeurs ne donnent pas lieu à une mise en concurrence des deux formes de critiques, c'est aussi parce que leur recours aux blogs est essentiellement motivé par une croyance dans la spécificité de l'apport des blogs par rapport à celui des critiques professionnels.

\section{Des capacités promotionnelles et un statut spécifiques}

Le pouvoir de prescription que les éditeurs reconnaissent aux blogs fonctionne selon des modalités différentes de la critique littéraire dans la presse et les médias audiovisuels. Ainsi, quand ils décrivent les effets de la visibilité d'une critique sur un blog littéraire, les éditeurs, quelle que soit leur taille, relèvent l'importance du "bouche à oreille ", c'est-à-dire le gain de lecteurs non pas en grand nombre en une seule fois, mais par un phénomène de réseau, par un "grignotage ", « les uns après les autres ", comme le formule l'un des responsables d'une petite maison d'édition nationale, ainsi que l'attachée de presse d'un grand éditeur national :

Moi j'en ai eu plein comme ça: «Putain, c'est génial, je connaissais pas, je vais passer ça à mon copain, il va transférer », etc. Et de fil en aiguille, voilà... Et ça, je pense que euh... ce truc par capillarité quoi, y a que les blogs ou Facebook ou... enfin c'est Internet quoi, typiquement !

17 Les éditeurs attribuent en outre aux blogs des qualités propices à la captation de nouveaux publics de lecteurs, moins sensibles à la critique littéraire dans la presse. Selon eux, si les blogs touchent d'autres publics, c'est parce que les blogueurs sont dotés de compétences spécifiques, distinctes de celles des critiques professionnels. Les blogs sont en effet situés du côté de la sensibilité, de la passion, de l'enthousiasme, et les critiques de presse du côté de l'argumentation et de la distance critique. Le responsable numérique d'un grand groupe d'édition souligne que «les médias traditionnels ont une tendance à objectiver un peu plus leurs propos, alors que les blogueurs sont plutôt sur la corde du sensible [...]. Donc c'est aussi une autre façon de parler au lecteur et à l'acheteur potentiel ». On retrouve cette idée dans les propos d'un autre enquêté:

Ça peut être horriblement mal écrit, mais ça, à la limite, c'est un blog, je m'en fiche de la qualité de l'écriture, hein. C'est pas des critiques. Je ne juge pas un blogueur par rapport à ce qu'il écrit dans le sens de la forme mais dans le sens du contenu. C'est-à-dire que s'il n'a pas d'enthousiasme dans sa lecture [...]

Donc, vous recherchez des gens qui...

... sont capables d'être des passeurs de livres. Et on passe un livre quand on est capable de lire correctement un livre et au moins de s'enthousiasmer. Même s'il ne le lit pas de la même façon que moi, peu importe! Mais qu'il soit en mesure de donner envie. Si je travaille avec des blogueurs, c'est pour qu'ils donnent envie aux autres de lire un bouquin. Si c'est totalement plan-plan, euh ... je travaillerai plus avec. L'idée c'est de trouver des... des passeurs. (Attachée de presse d'une maison d'édition de taille moyenne)

Cette enquêtée défend ainsi l'idée que les blogueurs sont capables de capter le lecteur par l'émotion et l'enthousiasme qu'ils transmettent et non pas en raison de compétences littéraires telles que le style d'écriture.

Si les blogueurs sont ainsi considérés comme capables de promouvoir des livres d'une autre manière, ils peuvent aussi apparaître comme de bons promoteurs de livres spécifiques, qui intéressent peu les médias traditionnels. Ainsi, certaines plateformes 
de critiques littéraires amateur ${ }^{10}$, qui travaillent notamment avec de grands éditeurs, font appel à des blogueurs pour promouvoir des littératures de genre (polar ou fantasy) ${ }^{11}$ et/ou plus difficiles à commercialiser (une partie de la littérature étrangère):

Il va y avoir des internautes très spécialisés sur un sujet et parfois beaucoup plus qu'un journaliste. [...] On a distribué des polars à des spécialistes de romans policiers qui en lisent de toute façon plus que n'en lirait n'importe quel journaliste spécialisé. (Responsable de plateforme)

Ainsi, du point de vue des éditeurs, les formes particulières de prescription permises par les blogs - prescription individualisée plutôt que prescription de masse, fondée sur la sensibilité plutôt que sur l'analyse, et portant sur la littérature spécialisée plutôt que sur la « littérature générale » (Olivera, 2007) - permettraient de rendre visibles d'autres livres et d'une autre manière. Les blogs ne concurrencent donc pas la critique professionnelle sur son propre terrain mais font émerger un autre type de critique.

Cette absence de concurrence transparaît enfin dans le statut que les éditeurs les plus importants - c'est-à-dire les plus à même d'influencer l'état des rapports de force entre les deux types de critiques - reconnaissent aux blogueurs : pour eux, il s'agit avant tout de lecteurs amateur et désintéressés plus que de critiques. L'indépendance financière, c'est-à-dire l'absence de rémunération des blogueurs pour les critiques qu'ils écrivent, est en effet un des critères qui, dans les entretiens avec les éditeurs moyens et grands, permet de distinguer les blogs et les médias traditionnels. Cette indépendance financière est étroitement liée à l'idée de l'indépendance de jugement, c'est-à-dire de la liberté pour le blogueur de choisir les livres qu'il souhaite chroniquer et d'écrire une critique négative. Les éditeurs jugent ainsi très négativement la tendance de certains blogs à la professionnalisation, synonyme de perte d'intégrité ${ }^{12}$ :

$\mathrm{Y}$ avait des blogueurs qui étaient très intègres et qui sont devenus beaucoup moins intègres. [...] Maintenant, ils sont aussi intègres que les médias traditionnels. [...] C'est-à-dire qu'au départ les blogueurs, ils disaient : «Je ne veux pas qu'on me paye pour faire une revue de presse ou pour parler d'un livre». Et puis on s'est vite rendu compte que les blogueurs, au final, ils faisaient tout le temps, tous, les mêmes maisons d'édition et au final, c'est qu'ils étaient bien récupérés par des maisons qui leur donnaient des livres, voire qui faisaient plus pour qu'ils parlent de leurs livres. (Responsable numérique d'un grand groupe d'édition)

Si les éditeurs «moyens » et "grands » interrogés s'intéressent aux blogs de lecteurs (en recourant à des plateformes de critiques littéraires amateur, en lisant les billets rédigés sur les livres qu'ils promeuvent, en leur envoyant gratuitement des livres, etc.), c'est donc avant tout parce que les blogueurs ne sont pas des professionnels. Bien que l'indépendance et le "désintéressement» (Bourdieu, 1993) - en tant que valeurs reconnues au sein du champ littéraire - assurent une certaine légitimité aux blogs, ils sont aussi des arguments permettant d'affirmer l'amateurisme des blogueurs, perçus comme des acteurs dotés d'une sorte d'innocence préservée des intérêts professionnels et qu'ils doivent à tout prix conserver. En d'autres termes, en accordant aux blogs cette indépendance, les éditeurs leur reconnaissent une qualité qui les relègue du côté de l'amateurisme.

Le statut de lecteurs amateur plus que de critiques conféré aux blogueurs par ces éditeurs tient enfin à l'appréciation qu'ils font de la qualité de leurs chroniques. L'un deux instaure une hiérarchie très claire entre les blogueurs et les médias classiques sur ce point, tout en la nuançant en partie (peut-être à des fins " diplomatiques ", en raison de ses contacts avec des blogueurs): 
Y a d'excellentes surprises !... [silence] Y a vraiment d'excellentes surprises ! Moi je tombe parfois sur des billets euh mais dignes des plus grands critiques littéraires. Mais c'est rare. Voilà ! Faut pas se voiler la face ... C'est très rare. (Attachée de presse d'un grand éditeur commercial) littéraires dans le cadre de leurs études secondaires (filière littéraire au lycée) et supérieures. Après avoir obtenu un master de gestion, Catherine a travaillé comme développeuse informatique et chef de projet puis elle a dû interrompre son activité professionnelle pour des raisons de santé. Laurence et George sont quant à elles titulaires d'un diplôme de lettres modernes (maîtrise pour la première, DEA pour la seconde). George est rédactrice en télétravail et corrige des examens blancs à domicile, Laurence dirige une association de promotion de la lecture. Éric Bonnargent (titulaire d'une maîtrise de philosophie et professeur certifié) est le seul à avoir converti ses compétences en intégrant le monde de la critique littéraire papier. Son activité de blogueur l'a en effet progressivement amené à chroniquer des livres aussi dans la presse $^{13}$. Le fait de tenir un blog a également eu des répercussions sur la trajectoire (ici proprement professionnelle) de Laurence, mais dans le domaine du livre en général, et non pas, comme Éric Bonnargent, dans celui de la critique littéraire.

29

Deux figures de blogueurs émergent de l'analyse de leurs pratiques et prises de positions, qu'ils les rapportent en entretien ou qu'elles s'objectivent dans leurs blogs : celle des «lecteurs amateur " qui ne cherchent pas à modifier les rapports de force 
existants et qui ne s'estiment pas compétents pour cela, et celle des « prétendants » qui espèrent, du fait de leur expertise, peser dans la définition de la littérature digne d'être promue.

Le choix d'analyser le contenu des critiques postées par les blogueurs, en complément des propos recueillis auprès d'eux en entretien, est motivé par l'idée que la variété des attitudes des blogueurs à l'égard de la critique professionnelle s'objective dans le degré de spécialisation de leurs blogs et dans les formes des commentaires écrits (plus ou moins «objectifs» et savants). Ces caractéristiques sont en effet tout autant la manifestation de compétences littéraires (liées notamment au niveau et à la nature du diplôme obtenu par les blogueurs) que celle de l'orientation qu'ils donnent à leur blog et, ainsi, du type de commentaire littéraire qu'ils pratiquent. De fait, il apparaît que le blogueur le plus impliqué dans une concurrence explicite vis-à-vis de la critique professionnelle est aussi celui qui a le blog le plus spécialisé et le plus savant. Parallèlement, Catherine et George sont les moins concernées par la question de la critique professionnelle et tiennent les blogs aux contenus les plus éloignés de ce type de critique ${ }^{14}$.

\section{Les lecteurs amateur}

La position de lecteur amateur est représentée par Catherine et George. Toutes deux ne s'attribuent ni réel pouvoir de promotion des livres ni capacité de concurrencer les critiques professionnels et elles se vivent davantage comme des lectrices dont les billets relèvent d'un autre registre que les critiques publiées dans les médias traditionnels. La place importante des critiques négatives ${ }^{15}$ dans les billets qu'elles postent témoigne tout d'abord du fait que leur objectif premier n'est pas de promouvoir des livres qu'elles ont appréciés. En entretien, George explique par exemple qu'elle rédige un billet sur tous les livres qu'elle lit : elle ne sélectionne donc pas les livres qu'elle aurait particulièrement envie de défendre. Ensuite, ces deux blogueuses ne situent pas leur activité sur le même plan que la critique professionnelle. George se défend ainsi de toute volonté de professionnalisation de son activité de critique : elle se définit comme une «lectrice» qui émet "des opinions" et déclare sur son blog que sa «seule ambition" est de "[s]e faire plaisir, d'écrire sur [s]es lectures» (10 décembre 2011). Catherine se définit aussi en entretien comme une « lectrice » qui « donne ses avis » et elle n'exprime pas, en entretien, le sentiment d'avoir un statut différent des lecteurs qui ne tiennent pas de blog ( C'est pas parce qu'on a un blog que les blogs sont plus légitimes que l'avis d'un lecteur qui n'a pas envie d'avoir un blog»). Elle accorde essentiellement à son blog une fonction de partage de goûts littéraires, sans s'attribuer un véritable pouvoir de prescription :

Est-ce que vous pensez que vous avez une certaine influence sur les lecteurs? Parler d'influence... euh... Je sais que oui j'ai des retours! On me dit : « Oui, j'ai lu le livre que tu as beaucoup aimé, moi aussi je l'ai aimé. »

Des gens qui grâce à vous sont amenés à lire peut-être davantage vous pensez?

Peut-être davantage ou qu'ils se reconnaissent un peu dans mon billet, dans ce que j'aime, dans mes choix de lecture. C'est comme moi, y a des blogs que je vais suivre plus parce que, voilà, j'ai plus d'affinités littéraires. (Catherine, auteure du blog Manon et les livres) celui des critiques professionnelles, dans la mesure où leur communication n'est pas 
dirigée vers le marché mais vers les autres lecteurs de la blogosphère. Ces deux blogueuses ne se considèrent donc pas comme des "prescriptrices", au sens de critiques ou d'experts chargés de certifier la valeur des œuvres (Lizé et al., 2014). Il est frappant de noter à quel point cette position revendiquée par ces blogueuses rejoint la position que leur attribuent la plupart des éditeurs. Ces manières de se définir rejoignent également le contenu de leurs blogs et des textes qu'elles y postent. En effet, s'ils sont principalement consacrés à la lecture et à la littérature, ces deux blogs ne sont pas centrés sur la seule critique littéraire et sur «l'expression d'une compétence spécialisée dans un domaine » (Cardon \& Delaunay-Téterel, 2006). Ils traitent d'une diversité de sujets, allant du compte rendu de leurs consommations culturelles variées à des considérations relatives à leur vie quotidienne. Le blog de Catherine mêle par exemple étroitement annotations personnelles et commentaires sur des ouvrages littéraires. Son blog est en effet intimement lié à sa maladie:

À 33 ans, je me suis retrouvée en invalidité à ne plus pouvoir travailler du jour au lendemain. J'ai dû faire le deuil d'une vie active, de plein de choses, et je me suis raccrochée à la lecture. Et au début, j'ai créé un blog [consacré à sa maladie]. [...] C'était vraiment pour changer le regard des gens sur le handicap et raconter ce que pouvait être la maladie. (Catherine, auteure du blog Manon et les livres)

Si, depuis deux ans et demi, Catherine se consacre « vraiment à la littérature » et si elle a fait disparaître l'allusion à la maladie qui apparaissait dans le premier nom de son blog, elle affiche toujours une rubrique consacrée à des informations médicales sur son blog actuel, dont l'adresse URL mentionne toujours sa maladie. Les liens vers les sites médicaux renvoient aux diverses pathologies dont souffre Catherine et donnent ainsi des indications sur sa vie personnelle. De plus, dans une rubrique de son blog, Catherine évoque en détail sa vie quotidienne, sa situation personnelle et familiale, comme l'atteste ce billet:

Même si les horoscopes m'annoncent une année pourrie au moins jusqu'en juin [...] et bien non, je ne vais pas demander une piqûre pour pachyderme et faire ainsi un gros dodo de plusieurs mois ou devenir casanière. Non, non, non!

2011 sera l'année de mes 40 ans (arghhh) mais comme m'a dit Fifille Ado number one: «les femmes sont à leur apogée à 40 ans et ne font pas de crise de la quarantaine » (et toc !) suivi de « mais te connaissant maman, tu vas bien trouver le moyen de nous en faire une ». Donc, affaire à suivre... (Site anonymisé Manon et les livres, billet du 31.12.2010, consulté le 06.08.2012)

Elle fait part de ses états d'âme, de ses émotions et de son ressenti, sur un mode plus ou moins ironique, en jouant sur la complicité avec les lecteurs du blog, perçus comme des pairs auxquels on confie par écrit ses enthousiasmes et ses soucis plutôt que comme des lecteurs auxquels on impose une autorité critique. George n'hésite pas non plus à faire part de considérations personnelles. Ces deux blogueuses revendiquent ainsi une forme d'accessibilité et de complicité avec les lecteurs, par les formes d'énonciation de soi et d'adresses au lecteur qui parsèment leurs blogs.

Le contenu de leurs billets sur les livres correspond lui aussi aux qualités recherchées par les éditeurs. La dimension subjective de la critique est ainsi très sensible dans les billets rédigés par George et Catherine. Cette dernière use par exemple abondamment de termes marquant la subjectivité, à commencer par l'emploi du pronom personnel " je », mobilisé dans chacune de ses critiques, et par un style non académique qui donne plutôt l'impression d'un langage parlé et d'une forme de spontanéité :

J'ai été captivée, j'ai vibré... du pur bonheur!!!!! (Site anonymisé Manon et les

livres, billet du 21.08.2011, consulté le 15.08.2012) 
J'ai refermé ce livre scotchée et il m’a fallu du temps pour reprendre pied avec la réalité. (Site anonymisé Manon et les livres, billet du 27.08.2011, consulté le 15.08.2012) formes d'énonciation de soi qui caractérisent certains blogs: "sur-emploi de la première personne, marques émotionnelles multiples, mélange d'oralisation et d'effets littéraires, multiplication des verbes exprimant des sensations, jugements à l'emportepièce, auto-ironie, exagérations de toute sorte, etc.» (Cardon \& Delaunay-Téterel, 2006: 34). Ce type d'évaluations "repose sur une subjectivité revendiquée», par opposition à l'« esthétique littéraire distanciée » (Béliard, 2014:108 et 112) affichée par certains blogueurs "prétendants». On retrouve cette distinction entre deux grandes catégories de critiques dans les contributions d'amateurs sur un site web de critiques cinématographiques, "avec d'un côté des critiques centrées sur le film (sur son contenu et sa forme) et de l'autre des critiques centrées sur la réception (ce que le film fait au spectateur)» (Beaudoin \& Pasquier, $2014: 141$ ).

\section{Les prétendants}

Éric Bonnargent et, dans une moindre mesure, Laurence sont deux blogueurs qui revendiquent des compétences littéraires susceptibles de placer leurs blogs en concurrence avec les instances de promotion et de jugement littéraires établies. Contrairement aux deux blogueuses précédentes, ils assument un rôle de promotion et de prescription des livres, en se tenant plus à distance de la critique négative. Par exemple, c'est un souci de promotion des ouvrages sur son blog qui conduit Éric Bonnargent à éviter de publier de telles critiques: «On ne fait pas de critiques négatives. [...] On préfère faire aimer des livres plutôt que de les descendre ! [...] On préfère inciter à lire plutôt qu'inciter à ne pas lire!». Certaines rubriques de L'Anagnoste témoignent également du rôle de promotion assumé par ce blog, comme c'est le cas des rubriques «Éditeurs sachant éditer » et " Auteurs sur les hauteurs », qui mettent en avant les éditeurs et les écrivains appréciés sur le blog. La croyance dans leur pouvoir de prescription est aussi relativement forte chez ces deux blogueurs. Ainsi, Laurence se compare à une bibliothécaire ou une libraire donnant des conseils à des lecteurs. Quant à Éric Bonnargent, il pense que son blog L'Anagnoste a contribué à faire connaître des petits éditeurs et des auteurs qu'il qualifie d'« exigeants ", n'ayant pas accès aux canaux classiques de reconnaissance.

Ces deux blogueurs pensent en outre pouvoir entrer en concurrence avec les instances traditionnellement chargées de promouvoir et d'évaluer les livres. C'est le cas tout d'abord vis-à-vis des grands prix littéraires. Laurence estime avoir un rôle de découverte et de repérage de titres de littérature grâce à son blog et au prix qu'il décerne, car les ouvrages primés sont ensuite consacrés par des prix littéraires reconnus :

Il y a tout juste deux ans, je découvrais et vous vantais les mérites du premier roman d'Estelle Nollet, On ne boit pas les rats kangourous. Roman qui quelques mois plus tard était finaliste du Prix Biblioblog 2010 et remportait le Prix Roblès 2010. (www.biblioblog.fr, billet du 16.08.2011, consulté le 10.09.2012)

Ce même rôle de découverte est conféré par Éric Bonnargent à son blog, dont les billets ont traité de livres qui ont ensuite reçu un grand prix littéraire, tel que le prix Médicis 
en littérature étrangère. Dans le même temps, Éric Bonnargent cultive un discours très virulent envers la plupart des grands prix.

De manière générale, ce dernier blogueur est celui qui se présente le plus explicitement comme un prescripteur et, de fait, comme un concurrent frontal de la critique professionnelle ${ }^{16}$. Une telle prise de position peut être éclairée par le fait qu'il exerce une activité de critique " amateur » sur un support papier, ce qui produit sans doute un effet d'autorisation à ses yeux et aux yeux d'autrui. Pour Éric Bonnargent, «l'éclosion des blogs vient incontestablement d'un mécontentement envers la critique traditionnelle $»^{17}$. En estimant que les blogueurs sont intervenus en réaction à la critique professionnelle, il se place avant tout lui-même en opposition à cette critique. Il pense ainsi que les commentaires de livres postés sur les «bons » blogs littéraires sont de meilleure qualité que ceux que l'on trouve dans la presse traditionnelle. Ces derniers auraient comme défauts d'être trop superficiels car ils doivent respecter des contraintes de taille précises, mais également peu objectifs du fait du manque d'indépendance des titres de presse vis-à-vis des grands groupes d'édition. Dès lors, il estime que les critiques des médias classiques ne sont pas bien placés pour juger de la qualité d'un livre alors que les blogueurs peuvent jouer ce rôle, y compris en " dénichant des talents ignorés de la presse écrite » ou en faisant connaître des auteurs avant que la critique professionnelle ne s'y intéresse : «On peut voir qu'un gars comme Roberto Bolaño, ce sont les blogs qui l'ont fait connaître. On en a parlé bien avant sur les blogs, avant que la presse s'en empare à cause de ce succès. »

41 La volonté de peser dans le processus de consécration des ouvrages de littérature s'observe également dans les normes d'écriture de ces "prétendants", qui se rapprochent de celles de la critique littéraire "experte ». Ainsi, Laurence et surtout Éric Bonnargent ne publient que des critiques d'ouvrages littéraires et des entretiens avec des auteurs et rejettent tout sujet extra littéraire et toute personnalisation du contenu de leur blog. Leurs compétences en matière d'analyse littéraire et de rédaction de comptes-rendus de lecture transparaissent aussi à la lecture de leurs billets. Si les blogs des «lecteurs amateur» se caractérisaient par une forte subjectivité, une spontanéité proche de l'oral et de la conversation, et par la place qu'y occupe l'expression des émotions (Andreevskaia et al., 2007), les billets d'Éric Bonnargent et de Laurence témoignent au contraire d'un rapport très savant et distancié aux œuvres critiquées. Leurs commentaires approfondis et analytiques montrent une attention formelle portée à la spécificité littéraire des textes et au travail d'écriture, qui s'appuie sur des savoirs de type linguistique, historique et littéraire. Au contraire, on a vu que les commentaires participatifs des deux autres blogueuses mettaient en relation l'œuvre lue avec des expériences vécues et se traduisaient le plus souvent par une forte implication subjective du blogueur (Renard, $2011: 13$ ).

Les dix critiques analysées parmi celles qu'Éric Bonnargent choisit de réaliser sur les livres qu'il apprécie particulièrement sont ainsi relativement longues, structurées en cinq à dix paragraphes avec introduction et conclusion. Comme sur le blog de Laurence, le registre de langue est soigné et les fautes d'orthographe ou de syntaxe sont rares. La part du commentaire critique est importante et ne se cantonne pas au résumé, qui comporte lui-même une dimension d'analyse de l'œuvre. Par exemple, à propos d'un roman de Claude Izner (Le Petit homme de l'opéra), Éric Bonnargent évoque la polyphonie et la diversité des registres de langue. Il évoque certains jeux sur la forme dans un roman d'Hélène Sturm (Pfff) : 
L'écriture d'Hélène Sturm, musicale et pétillante, est un vrai régal pour les amoureux des lettres : les jeux de mots et la métatextualité seront autant de défis à relever pour le lecteur attentif» (http://anagnoste.blogspot.fr, billet du 18.08.2011, consulté le 04.10.2012) par leurs aspirations, le rôle qu'ils se définissent dans l'univers littéraire ainsi que par le public auquel ils s'adressent. D'un côté on trouve le pôle des «lecteurs amateur ", qui se perçoivent avant tout comme des lecteurs s'adressant à d'autres lecteurs, et qui doutent de leur pouvoir de prescription. De l'autre, on trouve les « prétendants » qui se conçoivent comme ayant un rôle dans la consécration des titres de littérature, qui peuvent parfois s'affirmer comme concurrents de la critique professionnelle; de fait leurs prises de position sont davantage tournées vers les acteurs du champ littéraire. Cette diversité de la critique amateur en ligne a été observée sur d'autres terrains que le nôtre, dans des travaux qui analysent conjointement les propriétés des amateurs qui s'expriment sur le web et celles de leurs contributions. Il en ressort notamment que «l'espace des contributeurs est clivé entre des contributeurs authentiquement profanes et une élite [...] rédigeant des commentaires très largement inspirés de la critique experte » (Beauvisage et al., 2013 : 134). D’autres auteurs ont également mis au jour «deux modalités d'exercice de la critique» sur un site dédié aux critiques cinématographiques, avec d'un côté des «critiques centrées sur le film (sur son contenu et sa forme) et de l'autre des «critiques centrées sur la réception (ce que le film fait au spectateur)» (Pasquier et al., 2014: 62). La démarche du présent article consiste à entrer dans le détail des propriétés des amateurs qui publient ces contenus, montrant ainsi les liens entre les commentaires rédigés et les compétences acquises par les blogueurs au cours de leur trajectoire.

\section{Conclusion}

47 En combinant deux outils d'enquête (entretiens approfondis et étude détaillée du contenu des blogs), cet article interroge l'état de la concurrence entre critique professionnelle traditionnelle et blogs d'amateurs dans le domaine littéraire. La démarche a consisté à analyser de manière croisée les pratiques et les points de vue 
d'acteurs traditionnels du livre (les éditeurs, abordés ici de manière privilégiée ${ }^{18}$ ) et de blogueurs. L'enquête permet d'observer que la prise en compte des blogs dans le travail de promotion des éditeurs reste très limitée car ces derniers sont principalement tournés vers la critique «traditionnelle». Cela peut s'expliquer par les qualités restreintes et spécifiques qu'ils prêtent aux blogs, qui contribuent à reléguer ces derniers dans le domaine de l'amateurisme. Du côté des blogueurs, notre étude montre que ceux-ci se présentent rarement comme des concurrents de la critique professionnelle, se situant souvent sur un autre plan et se présentant avant tout comme des lecteurs. Le seul blogueur interrogé qui questionne la domination de la critique professionnelle revendique la supériorité des «blogs littéraires " par rapport à la critique professionnelle. Cette prise de position le place en concurrent de la critique professionnelle à la fois explicitement lors de notre entretien mais aussi implicitement dans le contenu de son blog.

Si cet article s'est attaché à la question de la concurrence entre critique professionnelle et critique amateur en ligne, une autre forme de concurrence, liée à cette première, mériterait aussi d'être étudiée : celle qui oppose les blogueurs amateur entre eux. Sur ce point, il semblerait que les blogueurs proches de la figure de «lecteurs amateur » se positionnent par rapport aux autres blogueurs amateur avant tout parce qu'ils sont mus par des enjeux propres à la blogosphère (par exemple, par la question de savoir quel blogueur amateur a le plus de visiteurs sur son blog). Les « prétendants » tendent quant à eux à prendre position par rapport aux autres blogueurs pour souligner ce qui les distingue de la majeure partie d'entre eux, qu'ils jugent moins compétents (parce qu'ils évoquent leur vie personnelle autant que les livres ou parce qu'ils se contentent d'impressions subjectives non argumentées). Ce faisant, ils adoptent un regard sur les blogs proche de celui des éditeurs, ce qui renforce le constat déjà avancé dans cet article de la proximité de leurs préoccupations avec celles des acteurs traditionnels de l'univers littéraire ${ }^{19}$.

Bien que le degré de « conversion » à la promotion en ligne soit moindre dans la filière du livre que dans d'autres filières culturelles (cinéma, jeux vidéo, musique) (Beuscart \& Mellet, 2012), les entretiens avec les éditeurs, mais aussi avec les écrivains, les libraires et les organisateurs de manifestations littéraires, témoignent de la diversité, si ce n'est de l'intensité, de leurs usages professionnels des blogs de lecteurs, comme partenaires promotionnels mais aussi comme outils de veille, sources d'information ou comme moyen d'assurer une médiation entre les livres et les lecteurs (lorsque les blogueurs sont sollicités pour participer à des manifestations littéraires par exemple). De plus, si ces professionnels expriment des doutes sur l'efficacité actuelle de la mise en visibilité des livres de littérature sur les blogs, plusieurs d'entre eux sont toutefois persuadés que ces espaces de critique en ligne vont jouer un rôle de plus en plus important à l'avenir. On peut considérer que cette croyance dans l'impact des blogs - qu'elle porte sur l'avenir, ou qu'elle se manifeste aujourd'hui par un usage de ces supports à côté des médias traditionnels et par une ambition de certains blogueurs à peser dans la lutte pour la reconnaissance littéraire - constitue un indicateur de l'influence, même limitée, qu'ont acquis ces espaces de critique amateur. 


\section{BIBLIOGRAPHIE}

ANDREEVSKAIA Alina, BERGLER Sabine \& URSEANU Monica (2007). « All Blogs Are Not Made Equal : Exploring Genre Differences in Sentiment Tagging of Blogs ", International Conference on Weblogs and Social Media [en ligne], mis en ligne le 26.03.2007, consulté le 02.09.2013. URL : http:// www.icwsm.org/papers/3-Andreevskaia-Bergler-Urseanu.pdf AURAY Nicolas \& MOREAU François (2012). «Introduction », Réseaux, 30 (175), pp. 9-18. BASTARD Irène, BOURREAU Marc, MAILLARD Sisley \& MOREAU François (2012). « De la visibilité à l'attention : les musiciens sur Internet ", Réseaux, 30 (175), pp. 19-42. BEAUDOUIN Valérie \& PASQUIER Dominique (2014). « Organisation et hiérarchisation des mondes de la critique amateur cinéphile », 32 (183), pp. 125-159.

BEAUVISAGE Thomas, BEUSCART Jean-Samuel, CARDON Vincent, MELLET Kevin \& TRESPEUCH Marie (2013). « Notes et avis des consommateurs sur le web. Les marchés à l'épreuve de l'évaluation profane », Réseaux, 31 (177), pp. 131-161.

BEAUVISAGE Thomas, BEUSCART Jean-Samuel, MELlET Kevin \& TRESPEUCH Marie (2014). « Une démocratisation du marché ? Notes et avis de consommateurs sur le Web dans le secteur de la restauration ", Réseaux, 32 (183), pp. 163-204.

BÉLIARD Anne-Sophie (2014). «Jeux croisés entre critique amateur et critique professionnelle dans les blogs de séries télévisées ", Réseaux, 32 (183), pp. 95-121.

BENHAMOU Françoise, MOUREAU Nathalie \& PELTIER Stéphanie (2012). « La longue marche vers une appropriation cognitive du Web : le cas de la bande dessinée », Réseaux, 30 (175), pp. 83-105. BEUSCART Jean-Samuel \& COURONNÉ Thomas (2009). « La distribution de la notoriété artistique en ligne ", Terrains \& travaux, 15, pp. 147-170.

BEUSCART Jean-Samuel \& MELLET Kevin (2012). Promouvoir les œuvres culturelles. Usages et efficacité de la publicité dans les industries culturelles, Paris, La Documentation française.

BOIS Géraldine, NENTWIG Anne-Cécile, SAUNIER Émilie \& VANHÉE Olivier (2013). Le rôle des blogs de lecteurs dans la mise en visibilité des livres de littérature : une analyse sociologique de l'activité des blogueurs et de la prise en compte des blogs par les acteurs traditionnels de l'univers littéraire, Rapport de recherche pour le Département des études, de la prospective et des statistiques du Ministère de la Culture et de la Communication.

BOUNIE Daniel, EANG Bora \& WAELBROECK Patrick (2010). « Marché Internet et réseaux physiques : comparaison des ventes de livres en France », Revue d'économie politique, 120 (1), pp. 141-162. BOURDIEU Pierre (1993). "Intérêt et désintéressement ", Cours au collège de France à la faculté d'Anthropologie et de Sociologie à l'Université Lumière Lyon 2, les 1er et 8 décembre 1988. BOURDIEU Pierre (1998). Les Règles de l'art. Genèse et structure du champ littéraire, Paris, Seuil [1992]. BOURDIEU Pierre (1999). «Une révolution conservatrice dans l'édition », Actes de la recherche en sciences sociales, 126 (1), pp. 3-28.

CARDON Dominique (2010). La Démocratie Internet. Promesses et limites, Paris, Seuil.

CARDON Dominique \& DELAUNAY-TÉTEREL Hélène (2006). « La production de soi comme technique relationnelle. Un essai de typologie des blogs par leurs publics », Réseaux, 24 (138), pp. 15-71. 
CARDON Vincent (2014). « Des chiffres et des lettres. Évaluation, expressions du jugement de qualité et hiérarchies sur le marché de l'hôtellerie », Réseaux, 32 (183), pp. 207-245.

COULEAu Christelle \& HeLlegouarch Pascale (dir.) (2010). Les Blogs : écriture d'un nouveau genre?, Paris, L'Harmattan.

FLICHY Patrice (2010). Le Sacre de l'amateur. Sociologie des passions ordinaires à l'ère numérique, Paris, Seuil.

LABORDE-MILAA Isabelle \& TEMMAR Malika (2006). « Légitimités énonciatives dans le discours littéraire médiatique : inscriptions subjectives et positions inégales ", Semen, $\mathrm{n}^{\circ} 22$ [en ligne], mis en ligne le 02.05.2007, consulté le 18.02.2013. URL : http://semen.revues.org/2832

LAHIRE Bernard (2006). La Condition littéraire. La double vie des écrivains, Paris, La Découverte.

LEGENDRE Bertrand \& ABENSOUR Corinne (2007). Les Petits éditeurs. Situations et perspectives, Paris, La Documentation française.

LIZÉ Wenceslas, NAUDIER Delphine \& SOFIO Séverine (dir.) (2014). Les Stratèges de la notoriété. Intermédiaires et consécration dans les univers artistiques, Paris, Éditions des archives contemporaines.

MOREAU François \& PELTIER Stéphanie (2011). « La diversité culturelle dans l'industrie du livre en France (2003-2007)», Culture études, 4, pp. 1-16.

NAUDIER Delphine (2002). «La fabrication de la croyance en la valeur littéraire », Sociologie de l'Art, OPuS, 4, pp. 37-66.

NAULIN Sidonie (2014). « La blogosphère culinaire. Cartographie d'un espace d'évaluation amateur ", Réseaux, 32 (183), pp. 31-62.

OLIVERA Philippe (2007). « Qu'est-ce que la "littérature générale” ? La culture lettrée au prisme du marché du livre de la première moitié du XXe siècle », Revue de synthèse, 128 (1-2), pp. 27-49.

PASQUIER Dominique (2014). « Les jugements profanes en ligne sous le regard des sciences sociales », Réseaux, 32 (183), pp. 9-25.

PASQUIER Dominique, BEAUDOUIN Valérie \& LEGON, Thomas (2014). « Moi, je lui donne 5/5. Paradoxes de la critique amateur en ligne, Paris, Presses de l'École des Mines.

PINCH Trevor \& KESLER Filip (2011). « How Aunt Ammy Gets Her Free Lunch : A Study of the TopThousand Customer Reviewers at Amazon.com », working paper [en ligne], mis en ligne en 2011, consulté le 22.03.2013. URL : http://lammgl.files.wordpress.com/2011/03/how-aunt-ammy-getsher-free-lunch-final.pdf

PINTO Louis (1991). « L'émoi, le mot, le moi », Actes de la recherche en sciences sociales, 88 (1), pp. 78-102.

RENARD Fanny (2011). Les Lycéens et la lecture, Rennes, Presses universitaires de Rennes. VERBOORD Marc (2010). « The Legitimacy of Book Critics in the Age of the Internet and Omnivorousness : Expert Critics, Internet Critics and Peer Critics in Flanders and the Netherlands ", European Sociological Review, 26 (6), pp. 623-637.

VERBOORD Marc (2014). « The impact of peer-produced criticism on cultural evaluation : A multilevel analysis of discourse employment in online and offline film reviews ", New Media \& Society, 16 (6), pp. 921-940.

WEBER Florence \& LAMY Yvon (1999), « Amateurs et professionnels », Genèses, 36 (1), pp. 2-5. 


\section{NOTES}

1. Le cinéma, les séries télévisées, la cuisine, etc. (Pasquier, 2014).

2. Si les salariés permanents des grands quotidiens sont sans aucun doute des " professionnels » au sens où ils retirent la majeure partie de leurs revenus de l'activité de critique, cette catégorie prend aussi en compte les pigistes peu rémunérés et les contributeurs exerçant un second métier, ces modalités d'exercice de la critique étant fréquentes dans le domaine littéraire (Naudier, 2002). À côté de la formation, la rémunération permet ainsi habituellement de distinguer les " professionnels» des "amateurs» (Weber \& Lamy, 1999, p. 3). Cela ne signifie pas que les blogueurs ne reçoivent aucune rétribution matérielle pour leur travail, celle-ci prenant le plus souvent la forme d'envois de livres gratuits par les éditeurs ou, parfois, de laissez-passer pour des salons du livre. La qualification de "critique professionnelle traditionnelle » renvoie aussi au haut degré d'institutionnalisation de ses supports (Verboord, 2010, 2014). L'opposition des catégories «critique professionnelle» et "critique amateur» répond aux besoins de démonstration de l'article. Comme tout raisonnement s'attachant en priorité à souligner ce qui distingue deux catégories, celui-ci tend à gommer l'hétérogénéité interne à chacune d'elles. Ainsi, on parlera de «la » critique littéraire professionnelle en se référant à l'idéal-type de la critique «savante » ou experte alors qu'en réalité, la critique professionnelle peut prendre des formes variées, notamment selon le type de support concerné, plus ou moins généraliste, ou selon le profil et la «légitimité énonciative » du journaliste (Laborde-Milaa \& Temmar, 2006). Cette dénomination s'appuie toutefois sur des enquêtes qui évoquent des normes et des pratiques plus courantes dans la «critique professionnelle» (Verboord, 2014 ; Pasquier et al., 2014). Quant au terme " amateurs ", il ne préjuge en rien du niveau de compétences littéraires des blogueurs ni de la force de leur investissement dans leur activité de critique. Comme l'étude sur laquelle est fondé cet article avait justement, parmi ses objectifs, celui de cerner ces niveaux de compétences et d'investissement (Bois et al., 2013), nous avons davantage les moyens de préciser la diversité interne au groupe des blogueurs que nous sommes en mesure de le faire pour celui des critiques professionnels.

3. Les quelques observations que nous avons pu faire à ce sujet montrent qu'une tendance à renvoyer les blogs à l'amateurisme côtoie une nécessité de protéger les frontières de la critique (et donc une crainte de se voir concurrencé). Par exemple, quand Pierre Assouline s'exprime sur les blogs de lecteurs sur son propre blog, il critique le manque de «savoir-faire » des blogueurs en leur donnant au mieux une place "à côté » de la critique traditionnelle mais il exprime aussi son souhait de "défendre" la place de la critique professionnelle contre de nouveaux prétendants. Assouline, Pierre (2007), «La critique littéraire entre le blog et l'enclume », La République des Livres (http://passouline.blog.lemonde.fr/2007/05/08/la-critique-litteraire-entrele-blog-et-lenclume/).

4. Notre classement en niveaux de « légitimité » renvoie aux travaux sociologiques sur l'édition et sur le "champ littéraire» (Bourdieu, 1999; Lahire, 2006). Sur la base de ces travaux, les éditeurs ont aussi été distingués selon leur taille (importance du catalogue liée à l'ancienneté de la maison, nombre de titres publiés annuellement, poids économique, nombre de salariés, appartenance ou non à un grand groupe d'édition) et selon l'étendue de leur distribution (nationale/régionale). Ainsi, le "grand groupe d'édition » dont il est question dans cet article compte parmi les vingt plus importants groupes d'édition français avec un chiffre d'affaire de plusieurs centaines de millions d'euros par an. Les deux "grandes " maisons d'édition " commerciales» de notre population ont quant à elles été créées il y a plus de soixante ans, comptent plusieurs milliers de titres à leurs catalogues, publient plusieurs dizaines d'ouvrages par an, emploient plusieurs dizaines de salariés et font partie de grands groupes d'édition. L'une d'elles a édité récemment un ouvrage vendu à près d'un million d'exemplaires tandis que la seconde annonce un chiffre d'affaire annuel de dix à quinze millions d'euros. La maison d'édition 
que nous qualifions de "moyenne " est au contraire une maison d'édition indépendante, de création un peu plus récente (une quarantaine d'années), elle compte un millier de titres à son catalogue, publie environ trente-cinq titres par an et emploie huit personnes. Les deux «petits éditeurs nationaux " de notre population sont quant à eux de création beaucoup plus récente (une dizaine d'années), comptent donc un nombre plus restreint de titres à leurs catalogues (une soixantaine), et publient annuellement une dizaine de titres. Ils emploient tous deux trois à cinq salariés permanents et sont distribués dans toute la France. Enfin, nous avons interrogé un «très petit éditeur régional » de création très récente (cinq ans au moment de l'enquête), avec un faible nombre de titres à son catalogue (moins d'une dizaine), une publication de deux à trois titres par an et une auto-distribution assurée par ses deux membres, sur un territoire restreint.

5. Nous avons respecté les souhaits d'anonymisation des blogueurs : prénom et nom véritables pour Éric Bonnargent, prénom véritable sans le nom de famille pour Laurence, pseudonyme utilisé sur le blog pour George et total anonymat (y compris pour le nom de son blog) pour Catherine.

6. Sur chacun des blogs, dix billets commentant un roman français contemporain ont été analysés.

7. Les listes qui recensent et sélectionnent les blogs de lecteurs sont rares et il nous a donc fallu faire un choix à partir de possibilités très limitées. Le site Wikio nous a semblé pertinent car il était bien connu des professionnels du livre que nous avons rencontrés. Quant à la liste de la BNF, elle avait pour avantage d'émaner d'une institution reconnue et visible, susceptible d'émettre des jugements éclairés relativement indépendants des enjeux des champs de l'édition et de la critique littéraire. En dehors de cette liste, d'autres sources d'information attestaient de la qualité reconnue aux deux blogs sélectionnés, notamment de la part «d'experts» et de professionnels de la critique littéraire. Tous deux sont ainsi mentionnés dans les sélections de blogs «littéraires» effectuées par Le Magazine des Livres (numéro spécial, avril-mai 2009). Biblioblog fait également partie des «blogs de critique littéraire» répertoriés sur le site Weblettres, «le portail de l'enseignement des lettres» (P. Lavergne, «Sélection de blogs de critique littéraire », site Weblettres, 13 novembre 2011, en ligne: http://www.weblettres.net/ sommaire.php? entree $=20$ \&rubrique $=134 ? \mathrm{em} \_\mathrm{x}=22$ ).

8. Certains grands éditeurs que nous avons contactés (mais finalement pas interrogés) ont cependant expliqué qu'ils ne recouraient pas aux blogs pour promouvoir leurs ouvrages. On peut penser qu'ayant un accès routinisé à la presse littéraire, ils ne perçoivent pas toujours l'intérêt de ce travail promotionnel en ligne.

9. Ces partenariats prennent généralement la forme d'un envoi gratuit et régulier d'un exemplaire d'un ouvrage à un blogueur afin que celui-ci puisse le lire et poster, s'il le souhaite, un commentaire du livre sur son blog.

10. Ces plateformes - payantes - sélectionnent à la place des éditeurs les blogs les plus adaptés à leur ligne éditoriale. Elles mettent ainsi en contact un éditeur et un (ou des) blogueur(s). L'éditeur envoie ensuite un exemplaire du livre qu'il souhaite promouvoir au blogueur mais celui-ci n'a pas obligation de rédiger un billet à son propos.

11. Valérie Beaudouin et Dominique Pasquier soulignent de même la place plus importante accordée aux films de genre dans les contributions d'internautes sur un site généraliste de critiques cinématographiques amateur, par rapport à la place qu'ils occupent dans la critique professionnelle (Beaudouin \& Pasquier, 2014 : 139).

12. Des jugements similaires ont été observés à propos des « top reviewers » d'Amazon (Pinch \& Kesler, 2011).

13. Éric Bonnargent a été contacté, via son blog, par les responsables du Magazine des Livres pour publier des critiques littéraires. Il ne publiait donc pas de critiques dans la presse avant la création de son blog et c'est pour cette raison que nous l'avons intégré dans notre population de 
blogueurs "amateur». De plus, au moment de l'entretien, son activité principale reste celle d'enseignant.

14. Ces quatre blogueurs ne lisent pas avec la même intensité la critique professionnelle, et ils mentionnent des supports et des titres différents. Éric Bonnargent lit peu de critiques littéraires en dehors de celles du Matricule des Anges et du Magazine des Livres. Il a arrêté de lire Le Magazine Littéraire et Le Monde des Livres (il porte par ailleurs un regard critique sur « les grands noms du Monde ou de Télérama»). Laurence est une faible consommatrice de critiques littéraires (elle lit Le Magazine Littéraire et le Monde des Livres "très occasionnellement " et ne s'intéresse pas aux émissions littéraires audio-visuelles). Catherine consulte régulièrement les articles de Télérama et elle est une adepte de l'émission de télévision La Grande Librairie, présentée par François Busnel, sur France 5. Elle évoque également le magazine Elle. George suit l'actualité littéraire dans quelques magazines (comme Lire) et dans certaines émissions à la radio et à la télévision (Le Masque et la Plume et Ça balance à Paris). Elle cite aussi Pierre Jourde comme critique qu'elle apprécie, ainsi que Gérard Collard parce qu'il ose les critiques négatives.

15. Sur un échantillon de dix billets publiés à propos de romans de littérature française sur les blogs étudiés, le blog de George contient trois billets globalement négatifs et deux autres pointant des défauts dans le roman commenté, le blog de Catherine contient trois billets faisant part de ses réserves.

16. Si Laurence émet beaucoup d'avis sur la critique professionnelle (bien plus que les deux autres blogueuses) et si elle remet parfois en cause radicalement cette critique comme le fait Éric Bonnargent, son attitude est plus ambivalente. En effet, elle ne perçoit pas les blogs comme susceptibles de concurrencer ces professionnels. Elle définit à plusieurs reprises les blogueurs comme des «lecteurs » et pense que leurs ambitions sont radicalement différentes de celles des professionnels : » On n'est pas dans la même cour ! [...] Nous, c'est pas notre métier, c'est une passion. On n'est pas sur le même registre ».

17. «Littérature \& Internet, Éric Bonnargent (alias Bartleby) vs Marc Villemain », Le Magazine des Livres, $\mathrm{n}^{\circ} 20$, novembre 2009.

18. Les résultats concernant les autres acteurs traditionnels de l'univers littéraire interrogés sont assez similaires, même si la nature de leurs pratiques et perceptions relatives aux blogs diffère selon leur fonction (écrivains, organisateurs de manifestations littéraires ou libraires). Pour plus de détail, voir Bois et al., 2013.

19. Cette distinction n'a rien de surprenant étant donné les critères selon lesquels ces différents blogs ont été sélectionnés: les blogs bien classés sur le site Wikio, comme ceux de George et Catherine, le sont surtout parce qu'ils sont cités par d'autres blogs, d'où une forte « orientation intra-blogosphérique » (Naulin, $2014:$ 45), alors que les deux autres blogueurs ont été repérés par d'autres acteurs de l'univers littéraire. Outre les jugements experts de la BNF, ils sont par ailleurs mentionnés dans des journaux ou revues littéraires (Éric Bonnargent l'a été par exemple dans Le Matricule des Anges), ils ont été invités à s'exprimer dans des salons du livre, etc.

\section{RÉSUMÉS}

Les blogs de lecteurs se sont développés à partir du début des années 2000. Ils ouvrent le champ des émetteurs de jugements publics sur les livres, jusqu'alors réservé à la presse et aux médias audiovisuels. À partir d'entretiens approfondis avec des éditeurs et des blogueurs et d'une analyse de contenu de quatre blogs de lecteurs différents, l'article propose de questionner l'état 
des rapports de force entre critiques professionnels et blogueurs amateur du point de vue de leur pouvoir de promotion. Ses résultats permettent de nuancer une vision idéalisée d'Internet comme dispositif qui ferait des blogueurs amateur de nouveaux agents déterminants de la promotion littéraire. L'étude montre en effet que, pour la plupart des éditeurs, la concurrence entre critique amateur et critique professionnelle est très limitée et, qu'au mieux, les blogs existent aux côtés de la critique professionnelle. Seuls les plus petits éditeurs - c'est-à-dire ceux dont les pratiques promotionnelles ne sont pas en mesure de bouleverser les rapports de force entre critiques professionnels et blogueurs amateur - considèrent ces derniers comme des concurrents potentiels des premiers. Dans un second temps, l'article montre que rares sont les blogueurs qui se positionnent comme des concurrents de la critique professionnelle.

Readers' blogs have emerged and spread on the Internet since the early 2000s. They extend the field of persons in a position to publicly judge books, beyond the printed press and the audiovisual media. Based on in-depth interviews with publishers and bloggers and on a content analysis of four different readers' blogs, this paper aims at assessing the balance of power between professional critics and amateur bloggers, from the point of view of their promotional force. Its results nuance the idealized view of the Internet according to which amateur bloggers would now have a decisive role in promoting literary books. The study shows that, for most of the publishers, the competition between amateur criticism and professional criticism is very limited. It appears that, at best, readers' blogs exist alongside professional critics. The small publishers are the only ones who consider that bloggers might rival with professional critics, but they are precisely among the publishers whose promotional activities are not able to overturn the balance of power between the former and the latter. Secondly, the paper shows that very few bloggers position themselves as competitors with professional critics.

\section{INDEX}

Mots-clés : Internet, critique littéraire, blogs de lecteurs, amateurs, concurrence, éditeurs Keywords : Internet, literary criticism, readers' blogs, amateurs, competition, publishers

\section{AUTEURS}

GÉRALDINE BOIS

Université de Lorraine, 2L2S

EMILIE SAUNIER

ENS Lyon, Centre Max Weber

\section{OLIVIER VANHÉE}

ENS Lyon, Centre Max Weber 